\title{
Eruptive xanthomas, lipemia retinalis in a lean young female patient with new onset diabetes mellitus and hypertriglyceredemia
}

\begin{abstract}
Eruptive xanthomas and lipemia Retinalis are a very rare finding which is pathognomonic of underlying Hypertriglyceridemia and Diabetes mellitus.

Both these clinical entities are an indication of underlying metabolic derangements, so prompt action in the form of life style modification and drug therapy is important to avoid the development of serious long term complications like atherosclerosis and pancreatitis. We present a rare case of Eruptive xanthomas and lipemia Retinalis in 16years old young lean female patient as the first presentation of new onset Diabetes mellitus and underlying hypertriglyceridemia.
\end{abstract}

Volume 8 Issue 6 - 2020

\author{
Asad Ullah,' Sobia Sabir Ali² \\ Department of Diabetes and Endocrinology, Lady Reading \\ Hospital Peshawar, Pakistan
}

\author{
Correspondence: Asad Ullah, Department of Diabetes \\ and Endocrinology, Lady Reading Hospital Peshawar, Pakistan \\ Peshawar, Pakistan, Tel +923449054220, \\ Email aujb149@gmail.com
}

Received: September 22, 2020 | Published: December 08, 2020

\section{Background}

Eruptive Xanthomas are pink erythematous papules with creamy centers that mostly affect the extensors body surfaces involving the thighs, buttocks, knee and elbow joints usually bilaterally and symmetrically. Eruptive xanthomas appear as a result of lipid rich macrophage deposition in the dermis.

Lipemia Retinalis is another rare finding, which occurs in patients with serum triglycerides level $\geq 1000 \mathrm{mg} / \mathrm{dl}$. The retinal vessels look milky white on ophthalmologic examination. This is because of chylomicron visualization in the retinal vessels. ${ }^{2}$

Hypertriglyceridemia may be because of underlying primary familial genetic defects or secondary due to Diabetes mellitus, Hypothyroidism, nephrotic syndrome, obesity or alcohol intake.,

The risk of developing Eruptive xanthomas and lipemia Retinalis increase exponentially if a patient is having both Hypertriglyceridemia and uncontrolled Diabetes mellitus in this 16 years old young lean patient, Eruptive xanthomas was the first presentation of underlying Hypertriglyceridemia and new onset Diabetes mellitus.

Keeping in mind that Eruptive xanthomas and Lipemia Retinalis is the manifestation of underlying hypertriglyceridemia and uncontrolled Diabetes mellitus, strict measures should be taken to control both these conditions with life style modifications and drug therapy in order to prevent the long term complications like generalized atherosclerosis and pancreatitis. ${ }^{4}$

\section{Case presentation}

Eruptive Xanthomas with Lipemia Retinalis as a result of Hypertriglyceridemia and uncontrolled Diabetes mellitus are not uncommon in adults, but there is scarce data in Pediatrics age group. ${ }^{2,5,6}$ We describe a case report of a young lean female with BMI of $19.6 \mathrm{~kg} / \mathrm{m}^{2}$, who developed bilaterally symmetrical pruritic eruptions involving her knees, thighs, buttocks and elbow joints, blurring of her vision along with polyuria, polydypypsia and nocturia for the last 4weeks.
The patient past medical and surgical history was not significant, although there was a strong family history of T2DM in her maternal and paternal family but both her mother and father were non Diabetics. There was no family history of lipid disorder. She has four siblings and all of them are well. She studies in a religious school and does not drink alcohol.

On examination, the patient was of average built with a BMI of $19.6 \mathrm{~kg} / \mathrm{m}^{2}$.She had erythematous pink papules with creamy centers involving her knee joints, thighs, buttocks and the elbow joints bilaterally and symmetrically. The eruptions were diagnosed by Dermatology colleagues as "Eruptive Xanthomas".

The patient was complaining of blurred vision, so opthalmology opinion was sought and she was diagnosed to have Lipemia retinalis.

\section{Investigations}

Her laboratory investigations were as follows:

Triglycerides $1320 \mathrm{mg} / \mathrm{dl}(<200 \mathrm{mg} / \mathrm{dl})$, Total Cholestrol $591 \mathrm{mg} /$ dl $(<200 \mathrm{mg} / \mathrm{dl})$, High density lipoprotein $15 \mathrm{mg} / \mathrm{dl}$,low density lipoprotein $39 \mathrm{mg} / \mathrm{dl}$, Random blood glucose $434 \mathrm{mg} / \mathrm{dl}$, Fasting blood glucose $390 \mathrm{mg} / \mathrm{dl}$, Glycated haemoglobin $9.5 \%$, Fasting C-Peptide level $2.89 \mathrm{ng} / \mathrm{ml}(0.9-7.1)$, Urine routine examination showed three plus glucose level.

Other laboratoty investigations like Thyroid stimulating Hormone, S Lipase, S Amylase, Urine for protein, S ketones, Arterial blood gases, Full blood count, urea/creatinine, S Electrolytes, Liver function tests, Hepatitis B and C viral profile were in the normal range.

Her Ultarsound abdomen showed Hepatomegaly with liver size of $18 \mathrm{~cm}$ and increased parenchymal echogenicity but no element of portal hypertension. Lipid electrophoresis and genetic analysis could not be done because of Financial issues.

\section{Treatment}

The patient was educated about low glycemic and extremly low fat diet along with life style modifications.

The patient was started on Basal Bolus regime insulin. 


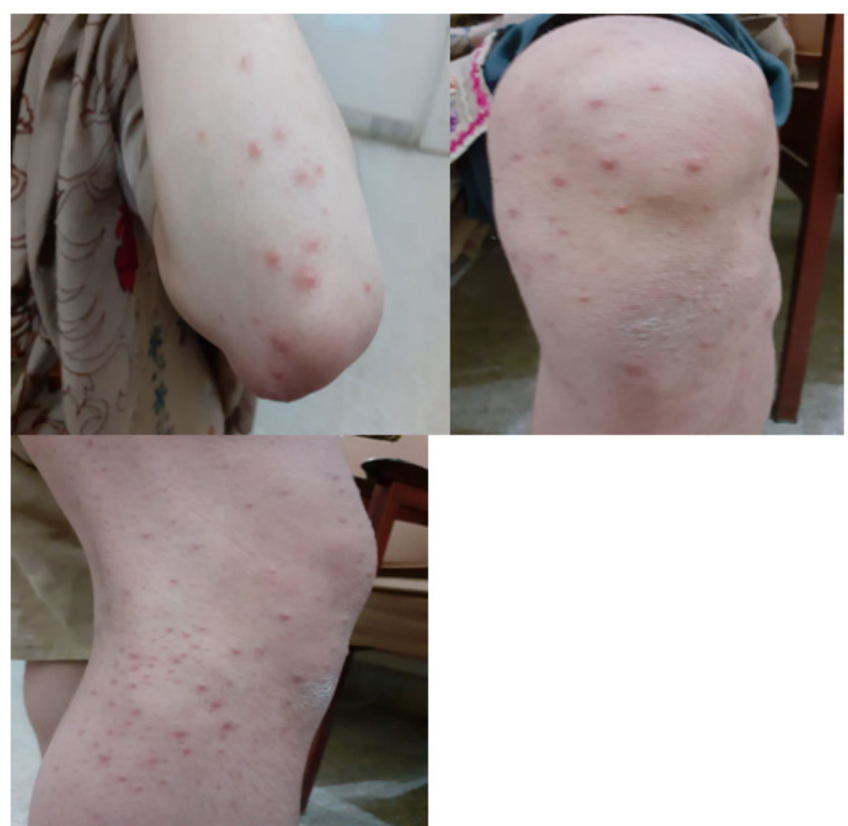

Figure I Erythematous pink papules with creamy centers involving her knee joints, thighs, buttocks and the elbow joints bilaterally and symmetrically.
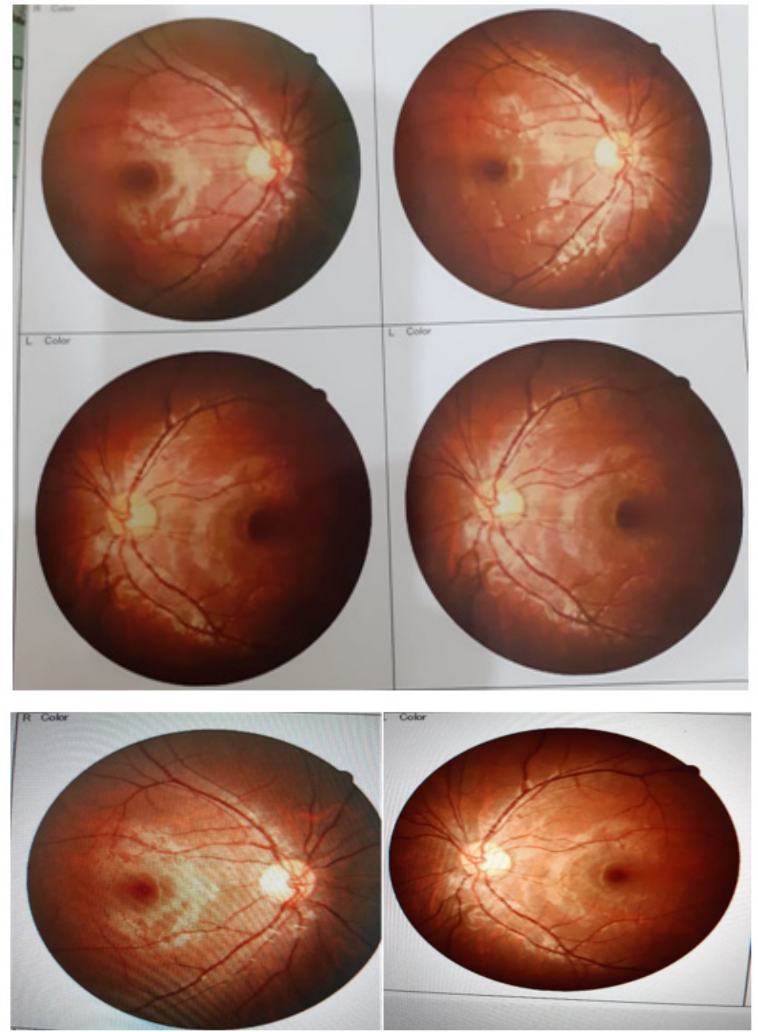

Figure 2 Lipemia retinalis.

She was given Inj Humulun R 16IU subcutanously three times a day before meals, Inj Lantus 12IU subcutanously at Bed time.

Metformin 750mg twice daily and Pioglitazone $15 \mathrm{mg}$ Once daily. Fenofibrate 200mg Once daily.

\section{Follow-up}

Because of the present COVID-19 pandemic, she could not be seen in the outpatient department for a follow up but a Tele-Medicine consultation was done, the patient lesions have resolved and her Blood glucose is well under the targets.

\section{Discussion}

Eruptive Xanthomas (EX) is erythematous pink papules with creamy centres that involve the extensor surfaces of the body extremities. These may be pruritic and tender to touch. They may exhibit the Koebner's phenomenon. Eruptive Xanthomas are associated with Hypertriglyceredemia specially occuring in type I, IV, and V (High concentrations of Chylomicrons and VLDL). There is a very high concentration of VLDL and Chylomicrons in these conditions.

EX can also occur in secondary hyperlipedemias like Diabetes mellitus, hypothyroidism, nephrotic syndrome, obesity and alcohol intake.

Lipemia Retinalis is a very rare presentation usually occurs at Triglyceride levels above $1000 \mathrm{mg} / \mathrm{dl}$. The retinal vessels give a milky appearance on opthalmologic examination. This is due to high chylomicron levels visualization in the retinal vessels.

In a nutshell, both Eruptive xanthomas and lipemia retinalis are pathognomonic of a severe underlying metabolic derangement like Hypertriglyceredemia and uncontrolled Diabetes mellitus and both these clinical entities usually resolves as the metabolic derangement are controlled with theraphy.

\section{Key learning points}

Eruptive xanthomas and lipemia Retinalis may point towards an underlying metabolic derangement specially Hypertriglyceredemia and Diabetes mellitus.

Eruptive xanthomas may be the first presentation of new onset Diabetes mellitus. Both eruptive xanthomas and Lipemia retinalis may resolve once the metabolic derangements are controlled with theraphy.

Eruptive xanthomas and lipemia retinalis associated with Hypertriglyceredemia and Diabetes mellitus should be treated in time in order to prevent the long term complications like generalized atherosclerosis and Pancreatitis.

\section{Acknowledgments}

None.

\section{Funding}

None.

\section{Conflicts of interest}

Authors declare that there is no Conflict of interest.

\section{References}

1. Binic I, Jankovic A. Eruptive xanthomas associated with diabetes mellitus. Chinese medical journal. 2009;122(17):2074-2075.

2. Rebecca G, Mark B. Eruptive xanthomas as a presenting feature of diabetes mellitus. Presented at British Society of Paediatric Endocrinology and Diabetes BSPED 2014, Winchester, UK: Endocrine Abstracts; 2014. 
3. Parker F. Xanthomas and hyperlipidemias. J Am Acad Dermatol. 1985;13:1-30.

4. Robson KJ, Piette WW. Cutaneous manifestations of systemic diseases. Med Clin North Am. 1998;82:1359-1379.

5. Berna S, Rabia OK, Seyyid BA, et al. First and only symptom of undiagnosed diabetes mellitus: eruptive xanthoma. BMJ Case Rep. 2015;2015:bcr2015212160
6. Zabeen B, Khaled Z, Nahar J, et al. Hypertriglyceridemia associated with eruptive xanthomas and lipemia retinalis in newly diagnosed diabetes mellitus. Mymensingh Med J. 2013;22(3):591-595. 